\title{
Study on metal interference correction method in mine TEM based on the fitting function
}

\author{
Shaohua Huang, Jiulong Cheng, Laifu Wen, Jin Zhou, Yun Long \\ College of Geoscience and Surveying Engineering China University of Mining \&Technology(Beijing) \\ Beijing, China \\ hshuang8200@163.com
}

\begin{abstract}
In order to improve the accuracy of mine transient electromagnetic(MTEM) detection results we carried out field detection experiments for the underground metal interference (tunneling machine, bolt and the anchor net),by analyzing the effects of interference, it turns out that these metallic facilities could produce strong transient electromagnetic response, there are some disciplinarians about metal interference in the transient electromagnetic response, the effect of interference is greater in the later period. We applied metal interference correction method based on fitting function for date processing, impaired and eliminated the low resistance false local anomaly, and the correction method improved the accuracy of results interpretation. The practical application shows that the tunneling machine has the most influential on the results of detection, the anchor net takes second place, minimum the bolt has.
\end{abstract}

Keywords-metal interference correction; mine transient electromagnetic; fitting function

\section{INTRODUCTION}

Mine transient electromagnetic method (MTEM) advanced detection technology could detect buried water conducting passages and predict water-bearing structures accurately and instantaneously, which is of great operational importance. Foreign geophysicists have a research history of the basic theory and applications of ground transient electromagnetic method for more than 50 years ${ }^{[1-4]}$, and the domestic research and application work on ground transient electromagnetic method also have made a number of valuable results in the past 30 years ${ }^{[5-8]}$. Due to the urgent need for coal mine water prevention and control, China University of Mining \& Technology introduced the transient electromagnetic method into coal mine roadway detection in 1998, and began to make use of SIROTEM-3F and terraTEM to do some MTEM experiments and application works. Since then, the application and technology of MTEM has obtained great improvement over the last decade ${ }^{[9-14]}$.

MTEM has become one of mature geophysical methods in roadway water-bearing structures detection because of its sensitive characteristics to low resistance object. But the actual condition in underground mine is complex, and the electromagnetic signal induced by metal objects in mining roadway such as tunneling machine, bolt, anchor net is usually stronger than that induced by water-bearing structures, and it does interfere with even cover the signal induced by water-

Natural Science Foundation of China (Grand No. 51574250); Specialized Research Fund for the Doctoral Program of Higher Education (No. 20120023110014) bearing area. Based on the similarity criterion of physical simulation, Yu Jingcun ${ }^{[10]}$ from China University of Mining \& Technology built mathematical model of correction affected by metallic facilities in roadway, and proposed the correction method and technology to metal interference induced by tunneling machine, bolt, anchor net etc.in roadway floor. According to the Maxwell equations and finite difference time domain method(FDTD), Zhou Xuan ${ }^{[11]}$ calculated and drew the full-space TEM respond characteristic curves under conditions of different metal layer thickness with non-uniform grid, offered a correction coefficient to correct the influence of metal support, and obtained some achievements. In this paper, according to the underground roadway experimental data, the TEM response characteristics affected by roadway metallic facilities such as machine, bolt, anchor net was analyzed and summarized, and the TEM advanced detection data correction technology was proposed based on the fitting function. Interference induced by low resistance false anomaly were corrected which helped improve the interpretation.

\section{Metal InTERfEREnCE CORRECTION Method}

\section{Metal Interference Correction Method Based on the Fitting Function}

By the experiments and the analysis of metal interference transient electromagnetic response characteristics, the existence of underground metal bodies had a certain impact on the actual detection results, in order to get more accurate measurement results, we need to carry certain correction on the actual detection data ${ }^{[15]}$, according to the analysis results, this paper started with transient electromagnetic induction electromotive force.

Calculation formula of mine transient electromagnetic method apparent resistivity ${ }^{[8]}$ :

$$
\rho_{\tau}=\beta \frac{\mu_{0}}{4 t \pi}\left[\frac{2 \mu_{0} S_{T} N_{T} S_{R} N_{R}}{5 t(V / I)}\right]^{2 / 3}
$$

In the formula, $\beta$ is the whole space influencing factors, $S_{T}$ and $S_{R}$ are respective areas of single turn transmitting and receiving loops; $N_{T}$ and $N_{R}$ are respective numbers of turns transmitting and receiving loops; The $V / I$ is normalization induction electromotive force after correction; $T$ induced the decay time of electromotive force. 
Under the same measurement working device, in the underground roadway, normalization induction electromotive force of without metal effect and metal effect are:

$$
\begin{aligned}
& A_{i}=V_{i} / I_{i} \\
& A_{i}^{\prime}=V_{i}^{\prime} / I_{i}^{\prime}
\end{aligned}
$$

The data can be obtained from multiple detection: $A_{1}, A_{1}^{\prime} ; A_{2}, A_{2}^{\prime} ; \cdots ; A_{m}^{\prime}, A_{m}^{\prime}$.

Order $B_{i}=A_{i} / A_{i}$, according to the comparative analysis of measured data in the underground roadway, the existence of metal interference increased the amplitude of transient electromagnetic induction electromotive force, slowed the attenuation down, there are certain rules between the ratio and time t. Suppose the given data $\left(t_{i}, B_{i}\right)(i=1,2, \ldots, m) . \Phi$ is function class and consisted of polynomial whose degree is no more than $n(n \leq m)$, we should work out $F_{n}(t)=\sum_{k=0} a_{k} t^{k} \in \Phi$, and make:

$$
I=\sum_{i=1}^{m}\left[F_{n}\left(t_{i}\right)-B_{i}\right]^{2}=\sum_{i=1}^{m}\left(\sum_{k=1}^{n} a_{k} t_{i}^{k}-B_{i}\right)^{2}=\min
$$

Figure out the Least squares fitting polynomial:

$$
F_{n}(t)=\sum_{k=1}^{n} a_{k} t^{k}
$$

So $F_{n}(t)$ is the fitting function we need, as the same time, $F_{n}(t)$ is also polynomial function, Select the proper degree according to accuracy requirements, get:

$$
\begin{gathered}
A_{i 0}=A_{i}{ }^{\prime} \cdot F_{n}(t) \\
\rho_{\tau}=6.32 \times 10^{-12} \times \beta\left(S_{T} N_{T}\right)^{2 / 3}\left(S_{R} N_{R}\right)^{2 / 3} A_{i 0}{ }^{-2 / 3} t^{5 / 3}
\end{gathered}
$$

$A_{i 0}$ is induction electromotive force after correction, according to the calculation formula of mine transient electromagnetic method apparent resistivity, we got the apparent resistivity after correction:

$$
\rho_{\tau}=6.32 \times 10^{-12} \times \beta\left(S_{T} N_{T}\right)^{2 / 3}\left(S_{R} N_{R}\right)^{2 / 3}\left[F_{n}(t) \cdot V_{i}^{\prime} / I_{i}^{\prime}\right]^{-2 / 3} t^{5 / 3}
$$

By this conclusion, according to the correction fitting function $-F_{n}(t)$ of different metal interference (tunneling machine, bolt, the anchor net),we should give the induced electromotive force correction value for each time window, then carry correction on the measurement data with metal interference, and we could get the apparent resistivity after correction, it makes the data processing results of the transient electromagnetic more accurate, the interpreting results of water-bearing structure in mining face would be more accurate and reliable.

\section{APPLICATION AND ANALYSIS}

After carrying out underground MTEM detection on a mine in Anhui Province, due to the existence of much metal bodies, such as tunneling machine, bolt, the anchor net, we found that the metal interference effected the measured data greatly, in order to get better results, we carried metal interference correction on measured data, it improved the accuracy of measured data and interpretation of the results. In the case of tunneling machine interference, we took metal interference correction method based on the fitting function to correct the measured data.

$100 \mathrm{~m}$, the apparent resistivity is relatively low, the lowest is $10 \Omega \cdot \mathrm{m}$, it reduced the existence of false anomaly.

The results above show that the interference correction for tunneling machine excluded the existence of false anomalies in actual detection effectively, reflected the actual apparent resistivity distribution in front of the heading face, it shows great agreement with later regular transient electromagnetic detection results and drilling results. The practical application proves the metal interference correction method based on fitting function is effective and correct.

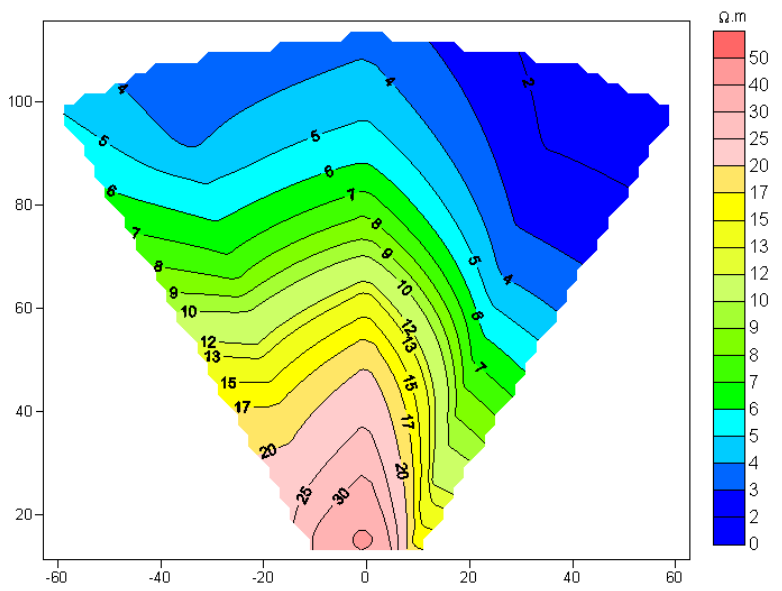

(a)The map of actual detection results with tunneling machine interference

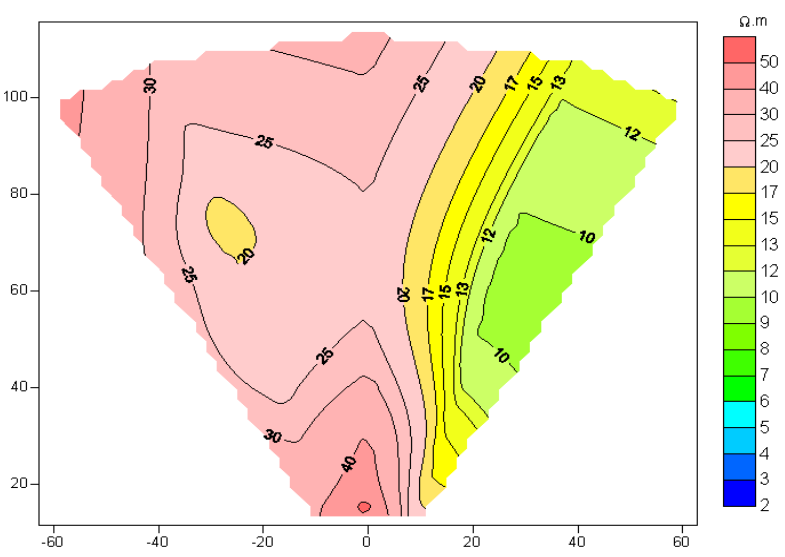

(b)The map of detection results without tunneling machine interference

Fig.1. Comparison map of tunneling machine interference correction

\section{CONCLUSION}

We researched and analyzed several common metal interference of transient electromagnetic response characteristics in mine transient electromagnetic method. The results show that the tunneling machine made the great impact 
on the detection results, anchor net made smaller impact and anchor rod with minimal impact.

The metal interference makes the amplitude of transient electromagnetic induction electromotive increases, making the attenuation slow, and making the apparent resistivity value relatively lower.

Through the metal interference correction method on the correction of measured data underground, weaken and eliminate the metal interference effect in measured data, reduce the false anomaly exists, making the corrected data is more beneficial to the accuracy, more applicable to the complicated mine conditions of instantaneous change electromagnetic data processing and interpretation, to improve the accuracy transient electromagnetic interpretation.

\section{ACKNOWLEDGMENT}

The research presented in this paper is supported by Natural Science Foundation of China (Grand No. 51574250), and Specialized Research Fund for the Doctoral Program of Higher Education (No. 20120023110014).

\section{REFERENCES}

[1] Wait James R. Transient electromagnetic propagation in a conducting medium. Geophysics, 1951, vol. 16, pp. 213-221.

[2] Morrison H. F., Phillips R. J., O'Brien D. P. Quantitative interpretation of transient electromagnetic fields over a layered half space. Geophysical prospecting, 1969, vol, 17, pp. 82-101.

[3] Lee Terry, Thomas Lindsay. The transient electromagnetic response of a polarizable sphere in a conducting half space. Geophysical prospecting, 1992, vol. 40, pp. 541-563.
[4] Wang Tsili, Hohmann Gerald W. A finite-difference, time-domain solution for three-dimensional electromagnetic modeling. Geophysics, 1993, vol. 58, pp. 797-809.

[5] Niu Zhilian. Time domain electromagnetic method. Changsha: Central South University press, 2007.

[6] Li Xiu, Theory and application of transient electromagnetic sounding. Xi'an: Shanxi science and Technology Press, 2002.

[7] Piao Huarong, Electromagnetic sounding principle. Beijing: Geological Publishing House, 1991.

[8] Yu Jingcun. The mine transient electromagnetic exploration. Xuzhou: China University of Mining and Technology press, 2007, pp. 66-67.

[9] Jiang Zhihai. Study on the mechanism and technology of advanced detection with transient electromagnetic method for roadway drivage face, China University of Mining and Technology, 2008.

[10] Yu Jingcun, Liu Shucai, Wang Yangzhou. Response characteristic of transient electromagnetic to metallic facilities in coal mines and the disposal technology. Journal of China coal society, 2008, vol. 33, pp. 1403-1407.

[11] Zhou Xuan, Liu Shucai, Chang Jianghao. Influences on Metal Support to Mine Transient Electromagnetic Detection and Correction Technology. Coal Science and Technology .2014, vol. 42, pp.101-104.

[12] Jiang Guoqing, Cheng Jiulong, Sun Xiaoyun, Chen Ding,Wang Haiyan. Optimized binary search algorithm of full space transient electromagnetic method all-time apparent resistivity. Journal of China Coal Society, 2014, vol. 39, pp. 2482-2488.

[13] Cheng Jiulong, Li Fei, Peng Suping, Sun Xiaoyun. Research progress and development direction on advanced detection in mine roadway working face using geophysical methods, Journal of China Coal Society, 2014, vol. 39, pp. 1742-1750.

[14] Cheng Jiulong, Chen Ding, Xue Guoqiang, Qiu Hao, Zhou Xiangtong. 2016. Synthetic aperture imaging in advanced detection of roadway using the mine transient electromagnetic method. Chinese J. Geophysics. (in Chinese), vol. 59, pp. 731-738

[15] Yu Lingang. Study on Processing of Early Transient Electromagnetic Signal. Chongqing University, 2013. 